\title{
How we are building Neuroanatomy and Behaviour for rigorous and open science
}

\author{
Shaun Yon-Seng Khoo $\oplus^{1,2, *}$ \\ ${ }^{1}$ Department of Pharmacology and Physiology, Faculty of Medicine, Université de Montréal, Montreal, Quebec, \\ Canada ${ }^{\prime}{ }^{\prime}$ \\ ${ }^{2}$ Episteme Health Inc., Melbourne, Victoria, Australia \\ *shaun.khoo@umontreal.ca
}

\begin{abstract}
Neuroanatomy and Behaviour was founded to be a journal for rigorous and open science. In 2021, all of the empirical papers published engaged in at least one open science practice, such as open data or open protocols. The papers published have been carefully reviewed by two experts, but may also be sent to additional specialist reviewers for specific tasks, such as checking references or statistical approaches. In 2021, Neuroanatomy and Behaviour reached a key milestone and was accepted into the Directory of Open Access Journals, the world's leading database of trustworthy open access journals. As we look towards 2022 , we will continue improving our publication processes and working to share quality neuroscience without financial barriers for authors or readers.
\end{abstract}

Key words: Open science; Open data; Peer review; Open access

\section{From the Editor}

Neuroanatomy and Behaviour was founded to be a journal for rigorous and open science. Our peer review process balances the confidentiality of traditional peer review to enable a fearless critique, while providing transparency with public review summaries. Our processes are designed to encourage authors to engage in reproducibility-enhancing practices, such as open data and preregistration.

\section{A Volume of Open Science}

In 2021, we published three review papers and four empirical papers. Tan and Kim reviewed the preclinical evidence for the role of the metabotropic glutamate 5 receptor in aversive learning [1]. McLemon and Chesworth discussed the potential for targeting the cannabinoid system in opioid addiction, considering both the preclinical effects on withdrawal and self-administration against some of the undesirable or off-target effects of cannabinoids [2]. Lay and Khoo reviewed the associative processes in addiction relapse models, arguing that cue-induced reinstatement is driven by a combination of conditioned reinforcement and Pavlovian-toInstrumental transfer [3].

It is pleasing to see that all of the empirical papers published in
Neuroanatomy and Behaviour this year have embraced openness in various ways. Didio and Casarotto shared the data from their survey on perceptions of reproducibility among neuroscientists via the Open Science Framework [4]. Burdakov and Karnani shared $40 \mathrm{~GB}$ of imaging data and supplementary video using Zenodo [5]. Cullity and colleagues also shared their behavioural and stereology data via Zenodo [6]. Finally, Maximino's study of the uptake ${ }_{2}$ monoamine transporter system was originally shared as a preprint on bioRxiv and is accompanied by both an open protocol and a dataset available on Github and Zenodo [7].

\section{Peer Review and Volunteers}

We also saw a mix of papers that were reviewed single-blind (anonymous reviewer/named author) and double-blind (anonymous reviewer/anonymous author). While our default process is to review papers single-blind, double-blind review can be requested by the authors and is required when one or more of the authors is on the committee of management of the publisher, Episteme Health Inc., or is a senior editor (e.g. Editor-in-Chief) of the journal.

Our reviewers helped us to evaluate papers in several ways. In addition to regular peer review, where an expert is asked to evaluate the whole paper, we also commissioned five specialist re- 
views from active researchers. Specialist reviews are optional additional reviews commissioned to examine specific features of a paper, such as whether references supported the point they were being cited for. Alternatively, a specialist reviewer may be asked to examine the appropriateness of the statistical approach. By supplementing regular review with specialist review, we have been able to provide an added level of rigour in the journal beyond what most journals are providing.

A noteworthy proportion of reviewers also embraced the option to sign their public review summaries, with 6/20 published reviews signed in 2021. In one case, all three reviewers chose to sign their reviews [4]. Consistent with prior studies [8,9], these results indicate to us that most reviewers value the option to remain anonymous, while others value the transparency that signing their reviews affords.

We have been very fortunate to have had so many scientistvolunteers taking on the tasks of reviewing, editing, and proofreading. On several occasions, we have been privileged to provide early career researchers with their first experience as a reviewer or editor. We are grateful to everyone for the time and expertise they shared with us and hope to work with them again soon, whether as authors or in editorial roles.

\section{Journal Development and Roadmap}

In 2021, the journal has had some major developments. Most importantly, Neuroanatomy and Behaviour was accepted into the Directory of Open Access Journals (DOAJ; https://doaj.org/toc/26521768). The DOAJ is the world's leading database of trustworthy open access journals. Inclusion in the DOAJ ensures that the journal appears as a fully open access journal in the Plan S Journal Check Tool (https://journalcheckertool.org). This is a key step towards establishing Neuroanatomy and Behaviour as a trustworthy source for behavioural neuroscience research. However, we need to maintain our publishing activity to continue to progress and enable us to apply for inclusion in PubMed Central ${ }^{\circledR}$.

We also made some changes to journal policies to better align our policies with best practice guidelines issued by the Committee on Publication Ethics and to ensure compliance with funder mandates. For example, this year we changed our license options from providing the Creative Commons Attribution license (CC BY) and its NonCommercial (CC BY-NC) variant to offering CC BY and its ShareAlike (CC BY-SA) variant. We have also made our willingness to accommodate requests for the CCo Public Domain Dedication more explicit. Creative Commons licensing is complicated [10-16], but these changes allow us to continue providing authors with licensing options while ensuring compliance with funder mandates such as Plan S.

As we seek out papers for 2022, we will continue to improve our publication processes, provide a quality experience for authors, reviewers, and editors, and advocate for an open access future that is free for both readers and authors.

\section{References}

1. Tan SZK, Kim JH. mGlu5: A double-edged sword for aversive learning related therapeutics. Neuroanatomy and Behaviour. 2021;3:e16. doi: 10.35430/nab.2021.e16.

2. McLemon E, Chesworth R. Cannabinoid treatment of opiate addiction. Neuroanatomy and Behaviour. 2021;3:e14. doi: 10.35430/nab.2021.e14.

3. Lay BPP, Khoo SYS. Associative processes in addiction relapse models: A review of their Pavlovian and instrumental mechanisms, history, and terminology. Neuroanatomy and Behaviour. 2021;3:e18. doi: 10.35430/nab.2021.e18.

4. Didio G, Casarotto P. The perception of reproducibility in a small cohort of scientists in Europe. Neuroanatomy and Behaviour. 2021;3:e20. doi: 10.35430/nab.2021.e20.

5. Burdakov D, Karnani $M$. Orexin neuron activity in mating mice - a pilot study. Neuroanatomy and Behaviour. 2021;3:e17. doi: 10.35430/nab.2021.e17.

6. Cullity ER, Guérin AA, Madsen HB, Perry CJ, Kim JH. Insular cortex dopamine 1 and 2 receptors in methamphetamine conditioned place preference and aversion: Age and sex differences. Neuroanatomy and Behaviour. 2021;3:e24. doi: 10.35430/nab.2021.e24

7. Maximino C. Decynium-22 affects behavior in the zebrafish light/dark test. Neuroanatomy and Behaviour. 2021;3:e21. doi: 10.35430/nab.2021.e21.

8. Bravo G, Grimaldo F, López-Iñesta E, Mehmani B, Squazzoni F. The effect of publishing peer review reports on referee behavior in five scholarly journals. Nature Communications. 2019;10(1):322. doi: 10.1038/s41467-018-08250-2.

9. Ross-Hellauer T, Deppe A, Schmidt B. Survey on open peer review: Attitudes and experience amongst editors, authors and reviewers. PLoS ONE. 2017;12(12):e0189311. doi: 10.1371/journal.pone.0189311.

10. Holl A. Response to "Is CC BY the best open access license?". Journal of Librarianship and Scholarly Communication. 2012;1(2):eP1055. doi: 10.7710/2162-3309.1055.

11. Graf K, Thatcher S. Point \& counterpoint: Is CC BY the best open access license? Journal of Librarianship and Scholarly Communication. 2012;1(1):eP1043. doi: 10.7710/21623309.1043.

12. Eve MP. In: Eve MP, editor. Open licensing. Cambridge: Cambridge University Press; 2014. p. 86-111. doi: 10.1017/CBO9781316161012.005.

13. Yi D. Is Creative Commons a panacea for managing digital humanities intellectual property rights? Information Technology and Libraries. 2019;38(3). doi: 10.6017/ital.v38i3.10714.

14. Mallalieu R. The elusive gold mine? The finer details of Creative Commons licences - and why they really matter. Insights: the UKSG journal. 2019;32(1):1. doi: 10.1629/uksg.448.

15. Gulley N. Creative Commons: Challenges and solutions for researchers; a publisher's perspective of copyright in an open access environment. Insights: the UKSG journal. 2013;26(2):168-173. doi: 10.1629/2048-7754.107.

16. Dörre DT. Current case law on Creative Commons licences. Journal of Intellectual Property Law \& Practice. 2015;10(4):310-312. doi: 10.1093/jiplp/jpv037.

\section{Copyright and License}

Copyright (C) 2021. Shaun Yon-Seng Khoo. Except where otherwise noted, the content of this article is licensed under a Creative Commons Attribution 4.0 International License. You are free to reuse or adapt this article for any purpose, provided appropriate acknowledgement is provided. For additional permissions, please contact the corresponding author. 